\title{
EDITORIAL
}

\section{EL SEXO DE LOS INDICADORES Y EL GENERO DE LAS DESIGUALDADES}

\author{
Concha Colomer Revuelta \\ Directora de los Observatorios del SNS y de Salud de la Mujer. Ministerio de Sanidad y Consumo.
}

A estas alturas del desarrollo de la salud pública y del sistema sanitario nadie duda de la importancia de los indicadores de salud y de los de enfermedad y mortalidad para planificar y evaluar políticas, programas y servicios de salud. Pero también sabemos que no siempre coincide la información disponible o razonablemente factible con la que se necesitaría ${ }^{1}$. Y que describir algunos aspectos de la salud y enfermedad de una población no es lo mismo que comprender sus causas ni que conocer, para actuar, cómo los determinantes sociales influyen en los resul$\operatorname{tados}^{2}$.

En el caso de las desigualdades entre mujeres y hombres la diferencia entre describirlas y comprenderlas es lo que ha motivado el título de este editorial.

Las cuestiones de género interesan de manera creciente, pero su estudio ha estado alejado de las ciencias de la salud, que se han ocupado fundamentalmente de la biología y, por tanto, de los aspectos de la salud ligados al sexo, no al género. Al igual que la clase social no existía para la medicina, tampoco existía el género, aunque ambos estén estrechamente ligados a la salud, pero desde el ámbito social. Quienes en el mundo sanitario se han interesado por estos temas han tenido que acudir a las fuentes de la sociología o del feminismo.
En España el interés de la salud pública por las desigualdades en salud por razón de género se inició hace ya unos años. En el seno de la Sociedad Española de Salud Pública y Administración Sanitaria (SESPAS) se impulsó un grupo de trabajo para investigarlas y visibilizarlas. Este grupo desarrolló un observatorio, espacios de debate en los congresos y la inclusión de las desigualdades de género en los informes SESPAS $^{3-5}$.

En el terreno de la investigación, una iniciativa importante ha sido la constitución de la Red de investigación en salud y género, financiada por el Instituto de Salud Carlos III (RISG) ${ }^{6}$, que ha supuesto un salto cualitativo hacia la investigación competitiva. Además, desde 2005 el Observatorio de Salud de la Mujer de la DG Agencia de Calidad del Ministerio de Sanidad y Consumo viene financiando una línea prioritaria de investigación en salud y género en las convocatorias de investigación del Instituto de Salud Carlos III $^{7}$.

Por otro lado, los sistemas de información en salud y del sistema sanitario están siendo revisados en el marco del Plan de Calidad para el $\mathrm{SNS}^{8}$ para que permitan, en primer lugar, desagregar los datos por sexo, algo que aunque parece básico todavía no está generalizado. En segundo lugar, deberán ser sensibles a las desigualdades de 
género incluyendo variables relacionadas con ellas, y permitir un tipo de análisis que las detecte y explique ${ }^{9-11}$. Estos planteamientos se refuerzan desde las políticas generales de igualdad, que vienen desarrollando los organismos de igualdad ${ }^{12}$ desde hace años, y se recogen especialmente en la nueva Ley orgánica 3/2007, de 22 de marzo, para la igualdad efectiva de mujeres y hombres ${ }^{13}$.

La mayor parte de la información sobre salud, enfermedades y sistemas sanitarios, muestra diferencias más o menos importantes o significativas entre hombres y mujeres cuando se desagrega por sexo. Pero para la mayoría de ellas no tenemos explicaciones, lo que aumenta la incertidumbre sobre la calidad de la información, sobre su validez para la planificación y sobre la efectividad y equidad de las actuaciones sanitarias. Vivimos un momento especial en este campo ya que las pruebas sobre las desigualdades en salud por género comienzan a ser visibles, pero su compresión es todavía deficiente. Incluso aspectos ya descritos hace años, como las desigualdades de género en relación con la atención a la cardiopatía isquémica ${ }^{14}$, siguen sin conocerse ni aceptarse por parte del sector sanitario que atiende estos problemas de salud.

Más allá de la mera descripción de la situación por sexo, la comprensión de las causas de las desigualdades por género no es fácil para el sector sanitario porque no está formado para hacer ese análisis interpretativo ni para actuar sobre sus causas, lo que supone establecer acciones que requieren el trabajo con sectores no sanitarios. Esto explicaría en parte las dificultades y, en algunos casos, resistencias ante estos temas en el sector sanitario.

Pero si se quiere utilizar la información para mejorar políticas, programas y servicios de salud, con el fin ultimo de mejorar la salud de la población, será necesario utilizar indicadores que no sólo sean fiables y sostenibles, sino también sensibles a las desigualdades de género.

Las actuaciones de salud, para ser equitativas, deben tener en cuenta su impacto diferencial sobre la salud de las mujeres y los hombres y, por lo tanto, su capacidad para incrementar o disminuir las desigualdades. Aunque las causas de estas desigualdades sean sociales, los estereotipos de género influyen en la manera de relacionarse las personas con los servicios de salud y el personal sanitario con las personas usuarias $^{15}$.

El sistema de salud debería partir de la idea de que, aunque es un derecho, la igualdad en la oferta de servicios no asegura la equidad en el acceso y la efectividad de los mismos en toda la población por igual. Es necesario reconocer que son necesarias acciones diferentes, lo que se llama "acciones afirmativas", pasar de la igualdad a la equidad, para atender las necesidades diversas de la población y actuar en consecuencia, si se quiere conseguir que los servicios no incrementen las desigualdades.

\section{BIBLIOGRAFÍA}

1. Castaño-López E, Plazaola-Castaño J, BolívarMuñoz J et al. Publicaciones sobre mujeres, salud y género en España (1990-2005). Rev Esp Salud Publica. 2006, 80 (6): 705-16.

2. Peiró R, Ramón N, Álvarez-Dardet C et al. Sensibilidad de género en la formulación de planes de salud en España: lo que pudo ser y no fue. Gac Sanit, 2004,.18, suppl. 2: 36-46.

3. SESPAS Grupo de Género y Salud. Disponible en http://genero.sespas.es/indice.html. Accedido 26 de Marzo de 2007.

4. La salud pública desde la perspectiva de género y clase social. La salud pública desde la perspectiva de género y clase social. Informe Sespas 2004. C Borrell, MMar García-Calvente y JV Martí-Boscà editores. Gac Sanit 2005. Disponible en http:// www.sespas.es/fr_inf.html. Accedido 26 de Marzo de 2007. 
5. Colomer Revuelta C, Peiró Pérez R. ¿Techos de cristal y escaleras resbaladizas?: Desigualdades de género y estrategias de cambio en SESPAS. Gac. Sanit. 2002;16 (4): 358-60.

6. Red de investigación en salud y género (RISG). Disponible en http://www.easp.es/redgenero/web/ esp/index.asp. Accedido 26 de Marzo de 2007.

7. Instituto de Salud Carlos III. Disponible en http://www.isciii.es/htdocs/presentacionyestructura/secretaria/ayudas_subvenciones_secretaria.jsp. Accedido 26 de marzo del 2007.

8. Ministerio de Sanidad y Consumo. Plan de Calidad para el SNS. Equidad/Salud y género. Madrid: Ministerio de Sanidad y Consumo; 2006. Disponible en http://www.msc.es/organizacion/ sns/planCalidadSNS/e02.htm. Accedido $26 \mathrm{de}$ marzo del 2007.

9. Rohlfs I, Borrell C, Anitua C, Artazcoz L, Colomer C, Escriba V, Garcia-Calvente M, Llacer A, Mazarrasa L, Pasarín M, Peiró R, Valls-Llobet C. La importancia de la perspectiva de género en las encuestas de salud. Gac Sanit. 2000 Mar-Apr; 14(2): 146-55.
10. García Gómez M, Montserrat y Castañeda López R, Rosario. Enfermedades profesionales declaradas en hombres y mujeres en España en 2004. Rev. Esp. Salud Publica, 2006, 80 (4): 349-360.

11. Informe salud y género 2005. Observatorio de salud de la mujer, Agencia de Calidad, Ministerio de Sanidad y Consumo. Disponible en http:// www.msc.es/organizacion/sns/planCalidadSNS/p df/equidad/InfomeSaludyGenero2005.pdf. Accedido 26 de Marzo de 2007.

12. Instituto de la Mujer. Disponible en http://www. mtas.es/mujer/. Accedido 26 de marzo del 2007.

13. Boletín Oficila del Estado. Ley orgánica 3/2007, de 22 de marzo, para la igualdad efectiva de mujeres y hombres. BOE núm 12611 de 23/03/07. Disponible en http://www.mtas.es/mujer/politicas/Ley_Igualdad.pdf. Accedido 26 de marzo del 2007.

14. Healy B. The Yentl syndrome. N Engl J Med 1991; 325: 221-5.

15. Velasco S, Ruiz MT, Álvarez-Dardet C. Modelos de atención a los síntomas somáticos sin causa orgánica: De los trastornos fisiopatológicos al malestar de las mujeres. Rev. Esp. Salud Publica, 2006, 80 (4): 317-33. 